(1)

CrossMark

\title{
Tips for success in pulmonary hypertension treatment: progress in isolating endothelial cells from pulmonary artery catheters
}

\author{
Andrea L. Frump ${ }^{1}$ and Tim Lahm ${ }^{1,2}$ \\ Affiliations: ${ }^{1}$ Division of Pulmonary, Critical Care, Sleep and Occupational Medicine, Dept of Medicine, Indiana \\ University School of Medicine, Indianapolis, IN, USA. ${ }^{2}$ Richard L. Roudebush VA Medical Center, Indiana \\ University, Indianapolis, IN, USA.
}

Correspondence: Andrea L. Frump, Walther Hall, C400, 980 W. Walnut St, Indianapolis, IN 46202, USA. E-mail: anfrumpQiu.edu

@ERSpublications

The paucity of patient-derived pulmonary cells has been a limiting factor in the study of pulmonary hypertension; however, new advances have the potential to increase scientific translatability and identify novel mechanisms driving early disease http://bit.ly/2U1AUEw

Cite this article as: Frump AL, Lahm T. Tips for success in pulmonary hypertension treatment: progress in isolating endothelial cells from pulmonary artery catheters. Eur Respir J 2020; 55: 2000122 [https://doi. org/10.1183/13993003.00122-2020].

\begin{abstract}
"The whole of science is nothing more than a refinement of everyday thinking." (Albert Einstein)
Pulmonary arterial hypertension (PAH) is a rare but deadly cardiopulmonary disease, which has an unclear and multifactorial pathobiology [1]. Unfortunately, despite availability of 13 Food and Drug Administration-approved therapeutic interventions, the 5-year survival rate is just 21-64\% [2]. An important clinical need exists to better understand the molecular mechanisms that lead to PAH development. In addition, a growing interest exists in the use of personalised medicine approaches for $\mathrm{PAH}$, since such a strategy is meritorious in other proliferative diseases, such as cancer $[3,4]$.

$\mathrm{PAH}$ is characterised by dysregulated vasoconstriction, muscularisation of small pulmonary arteries, and formation of obstructive plexiform lesions [5]. The exact initiating event(s) and early mechanisms driving $\mathrm{PAH}$ progression remain inconclusive and are an area of intense research interest. Unlike the field of cancer, efforts to address these questions in PAH have historically been hindered by lack of access to patient samples. In contrast to other pulmonary diseases, such as interstitial lung disease, inflammatory lung disease, lung cancer or acute lung injury states, lymph node biopsies, parenchymal biopsies or bronchoalveolar lavage are contraindicated or not useful in PAH [6]. Efforts focused on patient specimens therefore have centred on explanted lungs at the time of lung transplantation or on studying circulating cells such as peripheral blood mononuclear cells (PBMCs) or blood outgrowth endothelial cells (BOECs). However, isolating cells and tissues from explanted lungs limits access to those patients with end-stage $\mathrm{PAH}$. In addition, lung transplantation for PAH is infrequently performed [7]. PBMCs and BOECs, furthermore, are limited by their unclear relationship with the events in the pulmonary vasculature (PBMCs, BOECs), their unclear origin (BOECs) and the need to obtain large blood volumes (BOECs) [8-
\end{abstract}


11]. There is an unmet need for human pulmonary vascular cells isolated from living patients and at early stages of PAH to examine changes in cell function during disease progression and in response to therapies.

While the initiating pulmonary vascular injury is multifactorial and incompletely understood (likely a perfect storm of genetic and environmental factors), the integral contribution of pulmonary artery endothelial cells (PAECs), one of the major cell types comprising the pulmonary vasculature, to PAH pathology is evident. Two main theories describe how dysfunctional PAECs directly or indirectly contribute to disease progression. The first line of evidence posits that the endothelium exhibits disordered proliferation and angiogenic responses after pulmonary vascular injury [5]. Analysis of human PAH lung tissue suggests that the imbalance of these processes leads to formation of obliterative plexiform lesions (a hallmark of advanced PAH). Building on this finding, later evidence indicated that PAECs in plexiform lesions are of monoclonal origin (likely driven by somatic mutations in growth regulatory genes) [12] and that PAH PAECs exhibit chromosomal abnormalities that confer a growth advantage [13]. These changes are associated with apoptosis resistance [14]. A second theory posits that pulmonary vascular rarefaction observed in PAH is being driven by PAH PAEC-mediated accelerated and sustained apoptosis, rather than apoptosis resistance [15]. Importantly, dysfunctional PAECs spur on disease progression not only by mis-regulating the balance between growth and apoptosis, but also by affecting their microenvironment and the cells surrounding them. PAH PAECs accomplish this by releasing an abnormal secretome, inducing pulmonary artery smooth muscle cell hyperproliferation, fibroblast activation and immune cell recruitment, providing a disease-promoting feedback loop and culminating in pulmonary vascular dropout and formation of plexiform lesions [5, 16]. PAH PAECs clearly are an integral driver of PAH pathogenesis, but several critical questions remain: How does PAEC dysfunction progress during the course of PAH? Does PAEC apoptosis occur before PAEC apoptosis resistance arises? Is PAEC hyperproliferation a feature of all PAH patients or is this limited to specific patient subtypes and disease stages? What metabolic or epigenetic changes do PAH PAECs undergo? Which mechanisms drive early PAH progression? How do therapeutics affect the function of PAH PAECs?

In this issue of the European Respiratory Journal, VeNTETUOLo et al. [17] demonstrate feasibility of a technique that may begin to address these questions. A reproducible method to harvest viable PAECs from living PAH patients has been an ongoing objective in the field. While a ground-breaking method of isolating PAECs from the balloon at the tip of the pulmonary artery catheter (PAC) was first published several years ago by Pollet et al. [18], this method has not widely been adapted due to technical and logistical challenges. Ventetuolo et al. [17] built upon the work by Pollet et al. [18] and have developed a modified method for PAC-based PAEC isolation. The authors provide detailed, transparent procedural level details of their method and share what they have learned along the way. Of note, some details of the protocol differ from those published in the prior report (e.g. lack of trypsinisation, centrifugation, lysis, cell sorting), suggesting that PAC-based PAEC isolation is a "tricky business" and that protocols working at one institution may not be successful at another. The authors are to be commended for the level of detail they provide, which will certainly make it easier for investigators at other institutions to implement this technique. Aside from providing a detailed protocol, this report has several other strengths: first, the authors include a large variety of pulmonary hypertension ( $\mathrm{PH}$ ) phenotypes (not just $\mathrm{PAH}$ ), thus mimicking a real-world scenario, where many $\mathrm{PH}$ referrals have non-PAH $\mathrm{PH}$. Second, in some patients, several isolation attempts occurred throughout their disease course, thus providing details on the feasibility of serial PAEC assessments. Third, the authors provide the first evidence that it may be feasible to link PAEC function assessed ex vivo/in vitro with clinical parameters or disease type. Since right heart catheterisation is a fundamental diagnostic technique that often is repeated throughout a PAH (or even $\mathrm{PH}$ ) patient's disease progression, it could allow for collecting PAECs across repetitive samples at various disease stages.

Several findings of this study are notable: First, the authors successfully cultured PAECs from 19 of 49 PAC balloon tips, establishing a success rate of $39 \%$. Second, the investigators identified a positive correlation between successful PAEC culture and cardiac index and pulmonary vascular resistance, suggesting that more profound haemodynamic alterations are associated with a higher rate of successful PAEC isolation and culture. Third, the authors demonstrate that functional assays (investigating apoptosis, migration and tube formation) are feasible in isolated PAH PAECs and may provide clinically relevant phenotypic information. Interestingly, the patient-derived PAEC lines demonstrated substantial variability in terms of growth and functional assays. Whether this variability indeed is due to true biological differences between patients or due to technical factors remains to be determined. If there indeed are robust phenotypical differences between cells from different patients, PAEC ex vivo characterisation would represent a powerful new tool for diagnosis and patient phenotyping and possibly also for risk stratification and treatment selection, thus representing a significant step towards a personalised medicine approach. With the rise of the -omics area, there is also a tremendous potential for using such approaches 
on isolated PAH (or PH) PAECs. An exciting prospect is that PAH medications could be selected based on cellular characteristics, and that treatment responses could be evaluated directly and in real time. Such an ex vivo drug response assessment may even be helpful for non-PAH forms of $\mathrm{PH}$, where selected patient populations may still be responsive to treatment with $\mathrm{PAH}$-specific drugs $[19,20]$. In contrast to cells harvested from lung explants, such ex vivo interrogations would even be feasible in at-risk populations and early disease stages, and could also be repeated over time. In the current manuscript, PAEC culture from serial catheterisations was met with success in one patient.

Despite this step forward, several questions remain. This study did not attempt to link disease severity or subtype to PAEC function and was underpowered to do so. This will be valuable for future investigations, not only for $\mathrm{PAH}$ but also other groups of $\mathrm{PH}$, several of which do not have viable therapeutic interventions or biobanked tissue samples. Furthermore, PAECs from PAC balloon tips by default are isolated from proximal pulmonary arteries. $\mathrm{PAH}$, on the other hand, is a disease of distal vessels, raising the question of how relevant proximal PAECs are to disease development and progression. However, it has now been established that changes in the mechanical properties of the proximal pulmonary artery contribute to right ventricle dysfunction and distal pulmonary artery remodelling [21], possibly implying proximal PAECs as disease modifier. Studies of these cells may therefore identify new mechanisms and pathways driving PAH. However, at least some of the cultured cells could have been derived from central veins, the right heart, or circulating blood derived cells. While the authors performed preliminary studies to minimise this possibility, further investigations are needed to eliminate this scenario for sure. As omics-based approaches become more refined at detecting different cell populations within a single cell type, we may be able to discern differences between PAECs originating from different locations. Finally, the rate of successful isolation and culture was only $39 \%$. Since PAEC isolation and culture are labour- and time-intensive, significant resources are needed and would be "wasted" in $>50 \%$ of cases. Since successful culture was associated with more pronounced haemodynamic alterations, evaluation of earlier and milder disease stages could be challenging. Additional experience with the technique and technological advances will hopefully increase the yield.

Ultimately, studies of a larger sample size and reproducibility of the protocol by other scientists are needed. However, the work by VenTETUOLO et al. [17] is a significant step in answering if/how PAEC changes affect PAH pathogenesis and if they can be used for ex vivo diagnostics and drug testing, suggesting a huge potential for significant progress in the field.

Conflict of interest: A.L. Frump reports grants from Actelion, outside the submitted work. T. Lahm reports personal fees for consultancy from Actelion, personal fees for advisory board work and lectures from Bayer, provision of materials from Eli Lilly, outside the submitted work.

Support statement: This work was supported by the AHA Career Development Award 19CDAA34660173 (A.L. Frump), ALA Catalyst Award CA-629145 (A.L. Frump), Actelion/Entelligence Young Investigator Award (A.L. Frump), NIH 1R01HL144727-01A1 (T. Lahm), VA Merit Award 1I01BX002042-07 (T. Lahm) Funding information for this article has been deposited with the Crossref Funder Registry.

\section{References}

1 Humbert M, Guignabert C, Bonnet S, et al. Pathology and pathobiology of pulmonary hypertension: state of the art and research perspectives. Eur Respir J 2019; 53: 1801887.

2 McGoon MD, Benza RL, Escribano-Subias P, et al. Pulmonary arterial hypertension: epidemiology and registries. J Am Coll Cardiol 2013; 62: Suppl. 25, D51-D59.

3 Newman JH, Rich S, Abman SH, et al. Enhancing insights into pulmonary vascular disease through a precision medicine approach. A joint NHLBI-Cardiovascular Medical Research and Education Fund workshop report. Am J Respir Crit Care Med 2017; 195: 1661-1670.

4 Sitbon O, Gomberg-Maitland M, Granton J, et al. Clinical trial design and new therapies for pulmonary arterial hypertension. Eur Respir J 2019; 53: 1801908.

5 Humbert M, Morrell NW, Archer SL, et al. Cellular and molecular pathobiology of pulmonary arterial hypertension. J Am Coll Cardiol 2004; 43: Suppl. 12, S13-S24.

6 Frost A, Badesch D, Gibbs JSR, et al. Diagnosis of pulmonary hypertension. Eur Respir J 2019; 53: 1801904.

7 Hoeper MM, Benza RL, Corris $\mathrm{P}$, et al. Intensive care, right ventricular support and lung transplantation in patients with pulmonary hypertension. Eur Respir J 2019; 53: 1801906.

8 Geti I, Ormiston ML, Rouhani F, et al. A practical and efficient cellular substrate for the generation of induced pluripotent stem cells from adults: blood-derived endothelial progenitor cells. Stem Cells Transl Med 2012; 1: 855-865.

9 Groeneveld DJ, van Bekkum T, Dirven RJ, et al. Angiogenic characteristics of blood outgrowth endothelial cells from patients with von Willebrand disease. J Thromb Haemost 2015; 13: 1854-1866.

10 Balistreri CR, Buffa S, Pisano C, et al. Are endothelial progenitor cells the real solution for cardiovascular diseases? Focus on controversies and perspectives. Biomed Res Int 2015; 2015: 835934.

11 Chong MS, Ng WK, Chan JK. Concise review: endothelial progenitor cells in regenerative medicine: applications and challenges. Stem Cells Transl Med 2016; 5: 530-538. 
12 Yeager ME, Halley GR, Golpon HA, et al. Microsatellite instability of endothelial cell growth and apoptosis genes within plexiform lesions in primary pulmonary hypertension. Circ Res 2001; 88: e2-e11.

13 Aldred MA, Comhair SA, Varella-Garcia M, et al. Somatic chromosome abnormalities in the lungs of patients with pulmonary arterial hypertension. Am J Respir Crit Care Med 2010; 182: 1153-1160.

14 Masri FA, Xu W, Comhair SA, et al. Hyperproliferative apoptosis-resistant endothelial cells in idiopathic pulmonary arterial hypertension. Am J Physiol Lung Cell Mol Physiol 2007; 293: L548-L554.

15 Chaudhary KR, Taha M, Cadete VJ, et al. Proliferative versus degenerative paradigms in pulmonary arterial hypertension: have we put the cart before the horse? Circ Res 2017; 120: 1237-1239.

16 Humbert M, Montani D, Perros F, et al. Endothelial cell dysfunction and cross talk between endothelium and smooth muscle cells in pulmonary arterial hypertension. Vasc Pharmacol 2008; 49: 113-118.

17 Ventetuolo CE, Aliotta JM, Braza J, et al. Culture of pulmonary arterial endothelial cells from pulmonary artery catheter balloon tips: considerations for use in pulmonary vascular disease. Eur Respir J 2020; 55: 1901313.

18 Pollett JB, Benza RL, Murali S, et al. Harvest of pulmonary artery endothelial cells from patients undergoing right heart catheterization. J Heart Lung Transplant 2013; 32: 746-749.

19 Vachiéry J-L, Tedford RJ, Rosenkranz S, et al. Pulmonary hypertension due to left heart disease. Eur Respir J 2019; 53: 1801897.

20 Nathan SD, Barbera JA, Gaine SP, et al. Pulmonary hypertension in chronic lung disease and hypoxia. Eur Respir J 2019; 53: 1801914.

21 Scott D, Tan Y, Shandas R, et al. High pulsatility flow stimulates smooth muscle cell hypertrophy and contractile protein expression. Am J Physiol Lung Cell Mol Physiol 2013; 304: L70-L81. 